REVIEW ARTICLE

Four Principles Regarding an Effective Treatment of Aging

\title{
Marios Kyriazis*
}

\author{
National Gerontology Centre, Larnaca 6021, Cyprus
}

\begin{abstract}
The question whether aging is a disease or not, has been asked by many professionals who are involved in the study of age-related degeneration. However, not only an agreement on this remains elusive, but also effective clinical treatments against human aging have not been forthcoming. In this Opinion paper I suggest that the complexity involved in aging is such that we need to remodel our thinking to involve a much more 'systems-oriented' approach. I explore four main principles which should be employed by those who are working on finding treatments against agerelated degeneration. First, I discuss the problems encountered in translating laboratory research into effective therapies for humans. Second, I propose that a 'systems-thinking' method needs to be more extensively employed, instead of relying exclusively on the current reductionist one. Third, it is submitted that we must learn from the history of life-extension research, and not blindly follow contemporary paradigms, which may lead us into yet more 'dead ends' with regards to therapies. Finally, I suggest that, we may need to employ certain universal notions and use these in order to gain insights into the mechanics of a possible therapy against age-related degeneration. Examples may be the principle of hormesis, those of degeneracy, exaptation, and others from cybernetic or systems science domains. By using this four-pronged approach we liberate our thinking from the shackles of existing common mistakes and fallacies, and we open the way for a fresh approach that may lead us towards entirely new paradigms for providing clinically effective therapies against agerelated degeneration.
\end{abstract}

Keywords: Reductionism, systems thinking, translational research, age-related degeneration, life extension, hormesis.

\section{INTRODUCTION}

It is important to clarify at the outset that the paper does not only refer to treatments for common, established chronic degenerative clinical conditions (for instance Alzheimer's disease, Parkinson's disease or cataract), but it also refers to therapies or treatments, preventative or curative, that may be directed at the heart of the aging process itself (such as senolytics, genetic therapies, rejuvenation biotechnologies, or other approaches) [1] which could lower the risk of mortality as a function of age. In clinical terms, aging is defined as 'time-related dysfunction', and the overall ideal aim is to postpone this dysfunction indefinitely [2]. With this in mind, the paper will explore four main principles which should be considered by those involved in finding treatments for such age-related degeneration. The aim is not to elaborate on existing or emerging approaches to treat age-related degeneration, but to 'zoom out' of current thinking and examine new perspectives which may be useful in this respect.

\section{TRANSLATING LABORATORY FINDINGS INTO EFFECTIVE THERAPIES FOR HUMAN AGING}

Despite a large amount of laboratory research, effective therapies against aging at a clinical level remain elusive.

*Address correspondence to this author at the National Gerontology Centre, 6021 Larnaca, Cyprus; Tel 00357 97606424; E-mail: drmarios@live.it
With regards to aging, the difficulties in translating research performed on animals to effective clinical treatments for humans are notorious [3-5].

We are witnessing an unwarranted dependence on studying simplistic models of aging, and examining issues relating merely to a 'bench to bedside' principle. But in addition to this, there are other stages of the translational process that need to be overcome. The following is an outline of a progressive model of the translation process [6]:

Stage T0 - Prior to conducting the actual research there is a need to identify and describe the subject and potential of this basic research.

Stage T1 -This stage aims to bridge the gap between basic research in the laboratory and possible health applications. Strictly, this model refers simply to the suitability of a treatment in general terms.

Stage T2- At this next stage it is necessary to evaluate the clinical usefulness (if any) of the therapeutic application. There is a need to establish if this particular pharmaceutical product or therapeutic procedure achieves better results than other common existing therapies in clinical practice.
Stage T3 - If there is clinical usefulness, then it must be assessed within a wider, community-based practice. The therapy must be integrated into other existing therapies and 
we must ensure that it reaches the actual patient groups which may benefit from the therapy in question.

Stage T4- There must be an assessment to establish whether the overall health impact of the particular application or therapy at a population level is indeed positive. Any putative improvement must be shown to be measurable and considerable.

Stage T5- It may be necessary to consider another, perhaps final, stage which is time-related. Because aging is a continual process, we may also need to assess the persistent efficacy of the proposed treatment for all affected humans. This must remain unfailingly favourable in the long-term. The substantial and measurable health improvements of stage T4 must persist for an indefinite time-period [7].

Rejuvenation biotechnologies (which aim to 'restore youth', i.e. combat the aging process) and of anti-aging medicine in general, are, on the whole, still at stage T1. A specific treatment may indeed be effective in laboratory animals, but this treatment must be shown to make a persistent (not transient) positive health impact at the level of the human population (stage T5). Although each of the above stages may not be impossible to achieve, nevertheless in practice, the totality of the problems seen from a systemic thinking perspective makes the effectiveness of any rejuvenation intervention much less promising [8].

Therapies aimed at established diseases of aging or any other time-dependent loss of function, must not rely only on biomedicine but must take into account non-biomedical factors such as behavioural, social, cognitive, psychological and other environmental influences [9, 10].

With regards to aging, there is excessive reliance on another faulty logic, namely that research on animals may lead to significant benefits in humans. This logic is faulty because of a variety of reasons [11]. One of these reasons is that the health of human populations must be considered in association with our environment, society, culture and other nonmedical parameters. Human-specific phenotypes may be dissimilar to animal ones, and even mammal or higher ape phenotypes are different than those of humans - in some cases from $11 \%$ to $31 \%$ different [12]. It has been demonstrated that microRNAs in humans may have special functions which outline significant differences between humans and other animal species [13]. MicroRNAs are relevant in this respect because they reflect environmental and epigenetic effects such as societal influences on the DNA. In this example it is highlighted that research into animals may not necessarily translate into human clinical benefits, due to differences in the activity of microRNAs.

In addition, target groups of patients are likely to be over 60 with multiple co-morbidities and polypharmacy issues. Therefore, failures of translation are likely, when we ignore the physiological and social context of the aging process [14].

\section{EMPLOY A 'SYSTEMS THINKING' APPROACH INSTEAD OF A REDUCTIONIST ONE}

While reductionism is rife in the field of disruptive rejuvenation biotechnologies [15], there are encouraging signs that the number of academics and researchers who think in terms of 'magnification' (systems thinking) instead of 'miniaturization' (reductionism) is growing progressively [16]. In systems thinking we take into account the whole of the subject in question, and consider the impact of the environment on this subject. The problem in question is magnified, as if the researcher is 'zooming out' in order to examine the wider picture. On the contrary, in reductionist thinking, the problem is broken down into individual pieces, hoping to unravel systemic properties by studying these individual pieces. In fact, it is unlikely that only one approach is going to be helpful, but a combination of thinking methods should provide useful answers.

Reductionist models of aging fail to account for a number of concepts that are associated with real-life complexity. One such concept 'Non-additive Determinism, more commonly known as 'emergence' [17] where a system is not simply demarcated by the sum of all of its individual components seen in isolation, but there are additional properties that add another layer of complexity in the system's behaviour. In this situation, emergence specifies that simply analysing individual components of a system does not align with the behaviour of the system seen as a whole $[18,19]$.

In addition to considering the properties of the individual components of a system, we must also take into account how biological and social factors define the behaviour of an organism. This is the concept of 'Reciprocal Determinism' [18], where the behavior of the biological and social factors interact in a complex way leading to results which may be unnoticeable when we study separately biological or social elements. Instead, it is necessary to consider the combined effects of both the biological and the socio-cultural environment of the patient [3].

By considering the dynamics of the entire system (the organism in association with the environment the organism is in) we move to new and perhaps more promising domains which examine the relationship between genes and society (social genomics) $[20,21]$ and the relationship between genes and culture (gene-culture co-evolution) [22]. Social genomics, as the term suggests, examines the effects of social stimuli and environmental challenges upon our genome [23]. Cole [23] states that:

"Systems-level capabilities emerge from groups of individual, socially sensitive genomes... and ... transcriptional biofeedback (that) empirically optimizes individual well-being in the context of the unique genetic, geographic, historical, developmental, and social contexts...Studies of human social genomics are now clarifying which specific types of human genes are subject to social regulation and mapping the social signal transduction pathways that mediate these effects. The results of these analyses are shedding new light on the molecular basis for social influences on individual heath, the genomic basis for human thriving, and the metagenomic capabilities that emerge from networked communities of socially sensitive genomes".

Emerging concepts are demolishing the notion that effective antiaging therapies can be found in a reductionist model 
alone. Concepts such as Precision Medicine [24], and Molecular Pathological Epidemiology (MPE) [25] describe how disease may affect each patient in unique manner, and therefore it could be impossible to devise strategies that can affect such variations of pathological processes.

A relevant concept here is the 'Unique Disease Principle' [26]. This states that:

"Each individual patient exhibits unique disease profiles based on an interaction of genetic, epigenetic and other factors such as cell interactions, nutrition, lifestyle, microbial exposure, social and racial elements, age differences etc".

According to this principle it is necessary to deploy therapeutic interventions that are specific to each individual patient, something that may be impossible in clinical practice.

\section{LEARN FROM THE HISTORY OF LIFE SCIENCE RESEARCH}

'Those who do not learn history, are doomed to repeat it.' George Santayana.

The history of life extension (i.e. searching for a way to avoid aging and live a long, healthy life) reflects paradigms which are in accordance with the medical doctrines and the beliefs of each chronological period. Prescientific dogmas were ruled by mysticism, which necessitated the effort to influence the intervention of supernatural beings. Therefore, magic and animistic interventions were the main forms of treatment of aging and disease [27]. Narratives concerning aging and death were refined and elaborated by Greek and Roman physicians and were incorporated into religious, medical and social practices. These concepts enjoyed a revival during the Renaissance in a journey that was grounded in Alchemy, eventually reaching modern times when a more systematic inquiry lead us into modern biotechnological approaches. When favourite treatments of diseases where herbs and plants, the treatment of aging was also believed to be found in herbs and plants. When hormones where first discovered, the belief was that the secret of long life was to be found in hormones [28]. Thus, ideas and opinions about the aging process are influenced by the changing ideas and combined opinions of medicine, society, art and culture of each particular period [29].

Contemporary paradigms suggest that, as disease can, on the whole, be cured by pharmaceutical, genetic, and biotechnological interventions, it is logical to expect that aging too can be cured by such interventions. A representative example in this respect is that offered by the concept of rejuvenation biotechnologies, as advocated by the SENS (Strategies for Engineered Negligible Senescence) concept [30]. This reductionist and simplistic thinking has not resulted in any useful or clinically applicable therapy since its inception nearly 20 years ago, despite world-wide advocacy and millions of funding $[3,4]$.

We should refine our thinking to take into account the evolving social, cultural and behavioural narratives and environments. In other words, to consider contextual factors, to create a diversity of viewpoints, and to co-create solutions that involve the participation of stake holders (active involvement of patients). We should learn from such narratives and try to forecast what future medicine would be like, so that to adapt our thinking to these future models. Already we are witnessing the emergence of new ways of interpreting health [31] with the rise of precision medicine, and systems thinking. These lead to a lesser reliance on reductionism and on animal research which may not be suitably translated in human benefits with regards to aging. New effective approaches against aging may reflect entirely new thinking, based on a wider framework of therapies, involving both basic research and systems sciences, instead of the current overreliance on basic animal research alone $[32,33]$.

This is true not only with regards to actual treatment, but also with the way aging itself was considered, as a process. For two centuries, starting from the mid-1500s, the quest was to find remedies in order to extend lifespan of older people, so that to benefit from the wisdom of these older people for longer [34]. From the 1800s however, aging was depicted as a period of decrepitude, to be feared and avoided, and so it needed to be eliminated completely from humanity. I quote from the above paper:

\begin{abstract}
"While sharing little with the advocates of the early modern period, the recent anti-age movement clearly mirrors many of the beliefs of the longevity advocates of a century ago. Both groups not only see old age as a disease to be eradicated through injections and operations, but also argue that the old constitute an enormous economic burden. These beliefs reveal that the new anti-age movement, like its early $20^{\text {th }}$ century precursor, is based on more than simple hair dyes, hormones, or diet. Rather, their ideas and actions ultimately serve to marginalize the very process of growing old".
\end{abstract}

We need to devise a correct approach for treating agerelated degeneration, without repeating some of the errors and fallacies of the past [35]. It is important to learn from mistakes we made throughout history and apply our wisdom in order to develop more coherent theories that are closer to the truth. One such example is the Indispensable Soma Hypothesis [1], which is explained here: www.indispensablesoma.info. We must anticipate the future.

\section{EXPLOIT UNIVERSAL NOTIONS}

There are certain natural notions and principles that are valid across several domains, and that can be used in order to gain insights into the general mechanics of therapy. We need not reinvent the wheel. Instead, we may learn from already existing concepts which have not, as yet, been given due attention by researchers. Not only these principles may occur naturally and lead to a particular beneficial outcome, but they can also be used purposefully in order to enhance an intended outcome. The examples mentioned here highlight the fact that universal mechanisms may be useful in ways we may not be immediately aware. Such mechanisms may help us in our quest for dealing effectively with age-related degeneration. Examples of such principles or concepts are: 


\subsection{Biological Amplification}

This states that activation of one biological process at a local level, can have favourable consequences elsewhere in the body. An example of Biological Amplification is the deregulation of the function of luteinizing hormone which may then result in an associated increased risk of Alzheimer's disease [36]. A more well-known example is that testosterone is not only active in the sexual domain, but it also increases bioavailability of antioxidants, and it improves immune responsiveness [37]. Biological Amplification is encountered in the case of physical exercise which does not only have effects on the muscle, but it may affect cognitive health [38]. Therefore, it may be quite the case that by using an approach that is targeted at one organ, we may experience distant, amplified effects which need to be accounted for.

\subsection{Selective Reinforcement}

This is a general concept in cybernetics and cognitive sciences. It is based upon behaviour modification which seeks to increase the occurrence of certain desired events, and decrease the occurrence of certain undesirable events, with the result that only positive events are retained. This increases the degrees of freedom, adaptation abilities, and information-content of a process. This effect is also described as 'Operant Conditioning' in behavioural sciences. The addition of a reinforcing stimulus modifies the behaviour of the organism and makes it more likely that this particular behaviour will occur again in the future. The effect is also found in biology, where actions or effects that have beneficial influence on the survival or evolution of an organism, may be used again repeatedly, thus improving the overall fitness of the organism. This shares common frontiers with the concept of exaptation and of hormesis, described below.

\subsection{Exaptation}

During the process of adaptation of an organism or of a system, a character or property that was evolved for one purpose, may be 'exapted' in order to facilitate a new function in that organism or system. Basically, in exaptation, an existing structure gains a new function by reusing a mechanism that is already present. Once this (beneficial) function becomes operational in a system (including a biological one), and a change of context occurs, then there will be fast emergence of radical new functions. In this case the biological mechanisms necessary for this new function are already present but they need adapting to a new function. This mechanism highlights the importance of mechanisms that may be adaptive and that increase fitness (the ability to survive and function well) in biological systems. Such mechanisms may assist in the emergence of new functions which may be useful in affecting the rate of human aging. For instance, as our environment is becoming more cognitive-oriented (instead of physically-oriented), existing biological mechanisms may be exapted in order to accommodate the new relationship between us and our environment. Therefore, by the mere fact that our society is becoming more cognitive, this may have benefits against age-related degeneration via exaptation [39].

\subsection{Degeneracy}

Degeneracy is an unfortunate term, yet its importance is continually growing. It specifically refers to:

"The ability of alternate structural pathways to achieve similar functional outcomes in one context, or dissimilar functional outcomes in divergent contexts" [40].

It is an environmentally-dependant process, where a system recruits multiple pathways in order to achieve flexibility of function and adaptation [41]. What this means is that complex adaptive systems, just like the human body, have many dissimilar structures which can achieve the same end function, depending on the environment they are in [42-44]. Therefore, we see once again the important of contextualisation, the importance of considering the human organism not in isolation, but in association with its environment. The concept of degeneracy may be quite relevant in our search for mechanisms which may diminish disease and age-related dysfunction. For instance, it may open up possibilities for new mechanisms to operate which may help preserve the long-term function of somatic cells at the expense of germline cells, therefore leading to an increase in lifespan [1].

\subsection{Hormesis}

The case of hormesis is gaining popularity among scientific research [45]. Hormesis is a dose-response phenomenon, where a low dose of a given stressor may result in health benefits for the exposed organism, whereas a higher dose may result in damage and disease [46]. In hormesis, slight stressful challenges may up-regulate defence and repair pathways, with a subsequent overall improvement in function [47]. Hormesis is invoked when the challenging stimulus is within a certain optimum range [48-52].

The notion that biological processes are subjected to universal principles indicates that it may not be necessary to rely excessively on reductionism. Instead, considering a wider, more inclusive picture, the entire system may be modified as a whole, even if the action concentrates on one specific aspect of the biological process.

\section{CONCLUSION}

This review outlines the immense problems associated with the concept that age-related degeneration can be 'fixed' through a physical therapy. Material, item-based interventions are currently the basis of anti-aging medicine and of rejuvenation biotechnologies [53-58]. In order to continue searching for ways to diminish the impact of aging we need to abandon our excessive reliance on reductionism [2-4, 5963] and, instead, develop a more sophisticated approach based on systems thinking [64-68]. This is not to claim that reductionism has no place in modern research or therapy, but our thinking must evolve along more complex lines. Historical perspectives may assist us in highlighting areas of potential methodological errors. Our research must not examine the patient in isolation but must consider the effects of the environment on the patient [69-72]. In addition, we must consider translational issues that may slow down our progress. Technology is changing our society [39], and the effects of this must be studied in detail. Modern, complex 
concepts such as Molecular Pathological Epidemiology and Social Genomics [73-80], as well as insights gained from Precision Medicine [81-87], degeneracy [88, 89], exaptation $[90,91]$, and hormesis [92] may help us along our quest. By considering a comprehensive, sophisticated four-pronged approach we may be able to make considerable progress in diminishing the impact of age-related degeneration. The intention of this paper is not merely to discuss current or potential treatments against age-related degeneration, but to encourage the development of a wider and more encompassing perspective on aging.

\section{CONSENT FOR PUBLICATION}

Not applicable.

\section{CONFLICT OF INTEREST}

The authors declare no conflict of interest, financial or otherwise.

\section{ACKNOWLEDGEMENTS}

Declared none.

\section{REFERENCES}

[1] Kyriazis M. Neurons vs. Germline: A war of hormetic tradeoffs. Curr Aging Sci 2017; 10(4): 242-5.

[2] Kyriazis M. Editorial: Novel approaches to an old problem: Insights, theory and practice for eliminating aging. Curr Aging Sci 2014; 7(1): 1-2.

[3] Kyriazis M, Apostolides A. The fallacy of the longevity elixir: Negligible senescence may be achieved, but not by using something physical. Curr Aging Sci 2015; 8(3): 227-34.

[4] Kyriazis M. The impracticality of biomedical rejuvenation therapies: Translational and pharmacological barriers. Rejuvenat Res 2014; 17(4): 390-6.

[5] Callahan CM, Foroud T, Saykin AJ, et al. Translational research on aging: Clinical epidemiology as a bridge between the sciences. Translatl Res J Lab Clin Med 2014; 163(5): 439-45.

[6] Schully SD, Khoury MJ. What is translational genomics? An expanded research agenda for improving individual and population health. Appl Translatl Genom 2014; 3(4): 82-3.

[7] De Grey AD. Rejuvenation biotechnology: The industry emerges, but short-termism looms. Rejuvenat Res 2016; 19(3): 193-4.

[8] Kyriazis M. Editorial: Re-thinking ageing: A cross-disciplinary perspective (A New Era for Ageing). Mech Ageing Dev 2017; 163: 1.

[9] Heneghan C, Goldacre B, Mahtani KR. Why clinical trial outcomes fail to translate into benefits for patients. Trials 2017; 18: 122.

[10] Abernethy AP, Wheeler JL. True translational research: Bridging the three phases of translation through data and behavior. Translatl Behav Med 2011; 1(1): 26-30.

[11] Hackam DG. Translating animal research into clinical benefit. BMJ British Med J 2007; 334(7586): 163-4.

[12] $\mathrm{Hu} \mathrm{HY}$, Guo S, Xi J, et al. MicroRNA expression and regulation in human, chimpanzee, and macaque brains. PLoS Genet 2011; 7(10): e1002327.

[13] Dweep H, Georgiou GD, Gretz N, et al. CNVs-microRNAs Interactions Demonstrate unique characteristics in the human genome. An interspecies in silico analysis. PLoS One 2013; 8(12): e81204.

[14] Henney AM. The promise and challenge of personalized medicine: Aging populations, complex diseases, and unmet medical need. Croat Med J 2012; 53(3): 207-10.

[15] Zealley B, de Grey ADNJ. Commentary on some recent theses relevant to combating aging. Rejuvenat Res 2018; 21(1): 70-6.

[16] Flood RL. The relationship of 'systems thinking' to action research. Syst Pract Action Res 2010; 23: 269-84.
Necka EA, Cacioppo S, Cacioppo JT. Social neuroscience of the twenty-first century. The University of Chicago, Chicago, IL, USA, 2015.

[18] Cacioppo JT, Berntson. Social psychological contributions to the decade of the brain: Doctrine of multilevel analysis. Am Psychol 1992; 47: 1019-28

[19] Kyriazis M. Third phase science: Defining a novel model of research into human ageing. Front Biosci (Landmark Ed) 2017; 22: 982-90.

[20] Cole SW. Social regulation of human gene expression: Mechanisms and implications for public health. Am J Public Health 2013; 103(S1): S84-S92.

[21] Grewenb KM, Coffeya KA, Algoea SB, et al. A functional genomic perspective on human well-being. PNAS 2013; 110: 33.

[22] Richerson P, Boyd R, Henrich J. Gene-culture coevolution in the age of genomics. In the Light of Evolution: Volume IV: The Human Condition. National Academies Press: OpenBook, 2010.

[23] Cole SW. Human social genomics. PLoS Genet 2014; 10(8): e1004601.

[24] Mohler J, Najafi B, Fain M, et al. Precision medicine: A wider definition. J Am Geriatr Soc 2015; 63(9): 1971-2.

[25] Ogino S, Lochhead P, Chan AT. Molecular pathological epidemiology of epigenetics: Emerging integrative science to analyze environment, host, and disease. Mod Pathol 2013; 26(4): 465-84.

[26] Ogino S, Fuchs CS, Giovannucci E. How many molecular subtypes? Implications of the unique tumor principle in personalized medicine. Exp Rev Molecular Diagnost 2012; 12(6): 621-8.

[27] Bromley DB. The idea of ageing: An historical and psychological analysis. Compr Gerontol C 1988; 2(1): 30-41.

[28] Voronoff S, Greffes Testiculaires. 1923. Publisher: Librairie Octave Doin. ASIN B000JOOIA0.

[29] Peterson M, Rose CL. Historical antecedents of normative vs pathologic perspectives in aging. J Am Geriatr Soc 1982; 30(4): 289-94.

[30] De Grey A, Rae M. Ending Aging: The rejuvenation breakthroughs that could reverse human aging in our lifetime. 2007. New York, NY: St. Martin's Press. ISBN 0-312-36706-6.

[31] Huber M. How should we define health? BMJ 2011; 343: d4163.

[32] Naaldenberg J. Healthy aging in complex environments. 2011 Thesis, Wageningen University, Wageningen, NL. Availabl at http://edepot.wur.nl/169511.

[33] Clarke P, Nieuwenhuijsen R. Environments for healthy ageing: A critical review. Maturitas 2009; 64(1): 14-9.

[34] Haber C. Life extension and history: The continual search for the fountain of youth. J Gerontol A Biol Sci Med Sci 2004; 59(6): B515-22.

[35] Ritch A. History of geriatric medicine: From hippocrates to marjory warren. J R Coll Physicians Edinb 2012; 42(4): 368-74.

[36] Palm R, Chang J, Blair J, et al. Down-regulation of serum gonadotropins but not estrogen replacement improves cognition in aged-ovariectomized 3xTg AD female mice. J Neurochem 2014; 130(1), 115-25.

[37] Fanaei H, Karimian SM, Sadeghipour HR, et al. Testosterone enhances functional recovery after stroke through promotion of antioxidant defenses, BDNF levels and neurogenesis in male rats. Brain Res 2014; 1558: 74-83.

[38] Hogan CL, Mata J, Carstensen LL. Exercise holds immediate benefits for affect and cognition in younger and older adults. Psychol Aging 2013; 28(2): 587-94.

[39] Kyriazis M. Biological ageing and clinical consequences of modern technology. Biogerontology 2017; 18(4): 711-5.

[40] Mason PH. Degeneracy: Demystifying and destigmatizing a core concept in systems biology. Complexity 2015; 20(3): 12-21.

[41] Mason PH, Domínguez DJF, Winter GA. Hidden in plain view: Degeneracy in complex systems. Biosystems 2015; 128: 1-8.

[42] Cropper EC, Dacks AM, Weiss KR. Consequences of degeneracy in network function. Curr Opin Neurobiol 2016; 41: 62-7.

[43] Gally JA. Degeneracy and complexity in biological systems. Proc Nat Acad Sci 2001; 98(24): 13763-8.

[44] Whitacre JM. Degeneracy: A link between evolvability, robustness and complexity in biological systems. Theor Biol Med Modelling 2010; 7(6): 6.

[45] Calabrese EJ, Mattson MP. How does hormesis impact biology, toxicology, and medicine? NPJ Aging Mech Dis 2017; 3: 13.

[46] Calabrese EJ. Hormesis: From mainstream to therapy. J Cell Commun Signal 2014; 8(4): 289-91. 
[47] Rattan SIS, Le Bourg E, Eds. Hormesis in health and disease. Boca Raton. CRC Press. 2014.

[48] Pardon MC. Hormesis is applicable as a pro-healthy aging intervention in mammals and human beings. Dose-Response 2010; 8(1): 22-7.

[49] Martucci M, Ostan R, Biondi F, et al. Mediterranean diet and inflammaging within the hormesis paradigm. Nutr Rev 2017; 75(6): $442-55$.

[50] Calabrese EJ, Dhawan G, Kapoor R, et al. What is hormesis and its relevance to healthy aging and longevity? Biogerontology 2015; 16(6): 693-707.

[51] Kim B, Brandli A, Mitrofanis J, et al. Remote tissue conditioningAn emerging approach for inducing body-wide protection against diseases of ageing. Ageing Res Rev 2017; 37: 69-78.

[52] Bujarrabal A, Schumacher B. Hormesis running hot and cold. Cell Cycle 2016; 15(24): 3335-6.

[53] Longo VD, Antebi A, Bartke A, et al. Interventions to slow aging in humans: Are we ready? Aging Cell 2015; 14(4): 497-510.

[54] Liu GH, Ding Z, Izpisua BJC. iPSC technology to study human aging and aging-related disorders. Curr Opin Cell Biol 2012; 24(6): 765-74.

[55] De Magalhães JP. The scientific quest for lasting youth: Prospects for curing aging. Rejuvenat Res 2014; 17(5): 458-67.

[56] Yener IB, Cagin U, Yilmazer A. Cellular reprogramming: A new way to understand aging mechanisms. Wiley Interdiscip Rev Dev Biol 2018; 7(2). doi: 10.1002/wdev.308.

[57] Nurkovic J, Volarevic V, Lako M, et al. Aging of stem and progenitor cells: Mechanisms, impact on therapeutic potential, and rejuvenation. Rejuvenat Res 2016; 19(1): 3-12.

[58] Swan M. Translational antiaging research. Rejuvenation Res 2010; 13(1): 115-7.

[59] Weinberg BA, Marshall JL, Hartley M, et al. A paradigm shift from one-size-fits-all to tailor-made therapy for metastatic colorectal cancer. Clin Adv Hematol Oncol 2016; 14(2): 116-28.

[60] Davis OK, Rosenwaks Z. Personalized medicine or "one size fits all"? Fertil Steril 2014; 101(4): 922-3.

[61] Anonymous. Time to reject the "one size fits all" myth. Glob Adv Health Med 2014; 3(6): 5-6.

[62] Piazza JR, Charles ST, Luong G, et al. One size fits all? Applying theoretical predictions about age and emotional experience to people with functional disabilities. Psychol Aging 2015; 30(4): 930-9.

[63] Terrera GM, Brayne C, Matthews F. CC75C study collaboration group. One size fits all? Why we need more sophisticated analytical methods in the explanation of trajectories of cognition in older age and their potential risk factors. Int Psychogeriatr 2010; 22(2): 291-9.

[64] Marusic U, Grosprêtre S. Non-physical approaches to counteract age-related functional deterioration: Applications for rehabilitation and neural mechanisms. Eur J Sport Sci 2018; 20: 1-11.

[65] Nowak S, Neidhart J, Szendro IG, et al. Interaction analysis of longevity interventions using survival curves. Biology (Basel) 2018; 7(1): pii: E6.

[66] Kirkwood TB. Systems biology of ageing and longevity. Philos Trans R Soc Lond B Biol Sci 2011; 366(1561): 64-70.

[67] Roukos DH. Longevity with systems medicine? Epigenome, genome and environment interactions network. Epigenomics 2012; 4(2): 119-23.

[68] Cevenini E, Bellavista E, Tieri P, et al. Systems biology and longevity: An emerging approach to identify innovative anti-aging targets and strategies. Curr Pharm Des 2010; 16(7): 802-13.
[69] McMurphy T, Huang W, Queen NJ, et al. Implementation of environmental enrichment after middle age promotes healthy aging. Aging (Albany NY) 2018; 10(7): 1698-721.

[70] Bonfiglio T, Vergassola M, Olivero G, et al. Environmental training and synaptic functions in young and old brain: A presynaptic perspective. Curr Med Chem 2018; doi: 10.2174/0929867325666180228170450.

[71] Zuelsdorff ML, Koscik RL, Okonkwo OC, et al. Social support and verbal interaction are differentially associated with cognitive function in midlife and older age. Neuropsychol Dev Cogn B Aging Neuropsychol Cogn 2017; 15: 1-17.

[72] Sale A. A systematic look at environmental modulation and its impact in brain development. Trends Neurosci 2018; 41(1): 4-17.

[73] Zhang T, Tan H. Molecular pathological epidemiology. Zhonghua Liu Xing Bing Xue Za Zhi 2015; 36(7): 762-4.

[74] Nishi A, Kawachi I, Koenen KC, et al. Lifecourse epidemiology and molecular pathological epidemiology. Am J Prev Med 2015; 48(1): 116-9.

[75] Nishihara R, VanderWeele TJ, Shibuya K, et al. Molecular pathological epidemiology gives clues to paradoxical findings. Eur J Epidemiol 2015; 30(10): 1129-35.

[76] Burke W, Appelbaum P, Dame L, et al. The translational potential of research on the ethical, legal, and social implications of genomics. Genet Med 2015; 17(1): 12-20.

[77] Cardoso SD, Teles MC, Oliveira RF. Neurogenomic mechanisms of social plasticity. J Exp Biol 2015; 218(Pt 1): 140-9.

[78] Duster T. Social diversity in humans: Implications and hidden consequences for biological research. Cold Spring Harb Perspect Biol 2014; 6(5): a008482.

[79] Hood L, Balling R, Auffray C. Revolutionizing medicine in the $21^{\text {st }}$ century through systems approaches. Biotechnol J 2012; 7(8): 9921001

[80] Hood L. Systems biology and p4 medicine: Past, present, and future. Rambam Maimonides Med J 2013; 4(2): e0012.

[81] Wafi A, Mirnezami R. Translational omics: Future potential and current challenges in precision medicine. Methods 2018; 151: 3-11. Snyderman R. Personalized medicine 2014: Has healthcare been transformed? Per Med 2014; 11(4): 365-8.

[83] Zhou L, Wang K, Li Q, et al. Clinical proteomics-driven precision medicine for targeted cancer therapy: Current overview and future perspectives. Expert Rev Proteomics 2016; 13(4): 367-81.

[84] Schork NJ. Personalized medicine: Time for one-person trials. Nature 2015; 520(7549): 609-11.

[85] Smith AD. Proteomic advances hold promise for precision medicine. Am J Manag Care 2014; 20(5 Spec No.): E14.

[86] Empey PE. Pharmacogenomics to achieve precision medicine. Am J Health Syst Pharm 2016; 73(23): 1906-7.

[87] Shapiro SD. The promise of precision medicine for health systems. Am J Health Syst Pharm 2016; 73(23): 1907-8.

[88] Turgeon M, Lustig C, Meck WH. Cognitive aging and time perception: Roles of bayesian optimization and degeneracy. Front Aging Neurosci 2016; 8: 102

[89] Delignières D, Marmelat V. Degeneracy and long-range correlations. Chaos 2013; 23(4): 043109 .

[90] Larson G, Stephens PA, Tehrani JJ, et al. Exapting exaptation. Trends Ecol Evol 2013; 28(9): 497-8.

[91] Schulz A. Exaptation, adaptation, and evolutionary psychology. Hist Philos Life Sci 2013; 35(2): 193-212.

[92] Calabrese EJ. Post-conditioning hormesis creates a "subtraction to background" disease process: Biological, aging, and environmental risk assessment implications. J Cell Commun Signal 2018; 12(1): $31-4$ 